\title{
Array-based comparative genomic hybridization identifies a high frequency of copy number variations in patients with syndromic overgrowth
}

\author{
Valérie Malan ${ }^{\star}, 1$, Suzanne Chevallier ${ }^{1}$, Gwendoline Soler ${ }^{1}$, Christine Coubes ${ }^{2}$, Didier Lacombe ${ }^{3}$, \\ Laurent Pasquier ${ }^{4}$, Jean Soulier ${ }^{5}$, Nicole Morichon-Delvallez ${ }^{1}$, Catherine Turleau ${ }^{1}$, Arnold Munnich ${ }^{1}$, \\ Serge Romana ${ }^{1}$, Michel Vekemans ${ }^{1}$, Valérie Cormier-Daire ${ }^{1}$ and Laurence Colleaux ${ }^{1}$
}

\begin{abstract}
Overgrowth syndromes are a heterogeneous group of conditions including endocrine hormone disorders, several genetic syndromes and other disorders with unknown etiopathogenesis. Among genetic causes, chromosomal deletions and duplications such as dup(4)(p16.3), dup(15)(q26qter), del(9)(q22.32q22.33), del(22)(q13) and del(5)(q35) have been identified in patients with overgrowth. Most of them, however, remain undetectable using banding karyotype analysis. In this study, we report on the analysis using a 1-Mb resolution array-based comparative genomic hybridization (CGH) of 93 patients with either a recognizable overgrowth condition (ie, Sotos syndrome or Weaver syndrome) or an unclassified overgrowth syndrome. Five clinically relevant imbalances (three duplications and two deletions) were identified and the pathogenicity of two additional anomalies (one duplication and one deletion) is discussed. Altered segments ranged in size from 0.32 to $18.2 \mathrm{Mb}$, and no recurrent abnormality was identified. These results show that array-CGH provides a high diagnostic yield in patients with overgrowth syndromes and point to novel chromosomal regions associated with these conditions. Although chromosomal deletions are usually associated with growth retardation, we found that the majority of the imbalances detected in our patients are duplications. Besides their importance for diagnosis and genetic counseling, our results may allow to delineate new contiguous gene syndromes associated with overgrowth, pointing to new genes, the deregulation of which may be responsible for growth defect.
\end{abstract}

European Journal of Human Genetics (2010) 18, 227-232; doi:10.1038/ejhg.2009.162; published online 21 October 2009

Keywords: array-CGH; overgrowth disorders; oncogenes; chromosome imbalance; tumor-suppressor genes

\section{INTRODUCTION}

Overgrowth syndromes are a heterogeneous group of conditions characterized by either localized or generalized excessive growth for age and sex. Most overgrowth syndromes are associated with various anomalies, including an increased risk for neoplasia and/or cognitive impairment. The clinical importance of overgrowth syndromes was documented in the nineteenth century and conditions such as the Beckwith-Wiedemann syndrome (OMIM 130650), the Sotos syndrome (OMIM 117550), the Simpson-Golabi-Behmel syndrome (OMIM 312870) or the Bannayan-Riley-Ruvalcaba syndrome (OMIM 153480) were delineated by the 1960s. In contrast, the molecular cause of a handful of overgrowth syndromes has been elucidated only recently. Despite these recent advances, the diseasecausing mechanism remains unknown in $20-40 \%$ of patients, underlining the genetic heterogeneity of these disorders. Moreover, a large number of patients with overgrowth syndromes do not belong to any recognizable condition and remain unexplained.

Interestingly, several chromosomal duplications and deletions have been characterized in patients with overgrowth, suggesting that some still unclassified overgrowth syndromes may be caused by subtle genomic imbalanced rearrangements. Tall stature is observed in patients with dup $(4)(\mathrm{p} 16.3)$ likely because of the dosage effect of the fibroblast growth factor receptor gene $3 .{ }^{1}$ Trisomy of $15 \mathrm{q} 26 \mathrm{qter}$ is frequently associated with tall stature and mental retardation caused by duplication of the insulin-like growth factor 1 receptor gene.,3 Various types of NSD1 aberrations have been identified in patients with Sotos syndrome, including $5 \mathrm{q} 35$ microdeletion. ${ }^{4}$ Interstitial deletions at 9q22.32q22.33 have been detected in two patients with macrocephaly, overgrowth and trigonocephaly. ${ }^{5}$ Finally, $\operatorname{del}(22)(\mathrm{q} 13)$ has also been associated with overgrowth and macrocephaly. ${ }^{6}$ These data strongly support the hypothesis that studying chromosomal aberrations in relation to overgrowth using sensitive tools may lead to the identification of a significant number of cryptic chromosomal anomalies.

In this study, we report the application of genome-wide array-based comparative genomic hybridization $(\mathrm{CGH})$ at a $1-\mathrm{Mb}$ resolution to the study of 93 patients with syndromic overgrowth. In addition to their clinical relevance, our results emphasize the importance of a chromosomal imbalance in the etiology of these conditions and may help to identify novel genes involved in growth anomalies.

${ }^{1}$ Departement de Génétique et INSERM U781, Université Paris Descartes, Hôpital Necker Enfants-Malades, Paris, France; ${ }^{2}$ Hôpital Armand de Villeneuve, Service de Génétique Médicale, Montpellier, France; ${ }^{3}$ Hôpital Pellegrin, Service de Génétique Médicale, Bordeaux, France; ${ }^{4}$ Hôpital Sud, Service de Génétique Médicale, Rennes, France; ${ }^{5}$ Hôpital Saint-Louis, Laboratoire d'Hématologie, Paris, France

${ }^{*}$ Correspondence: Dr V Malan, Département de Génétique et INSERM U781, Hôpital Necker-Enfants Malades, 149 rue de Sèvres, 75015 Paris, France. Tel: +33 144494983 ; Fax: +33 1444904 17; E-mail: valerie.malan@nck.aphp.fr

Received 14 April 2009; revised 16 July 2009; accepted 11 August 2009; published online 21 October 2009 


\section{MATERIALS AND METHODS}

\section{Subjects}

We recruited 93 patients born to unrelated parents who presented with unexplained syndromic overgrowth. All of them presented with (1) height $>95$ th percentile and/or (2) occipito-fronto circumference $>95$ th percentile and (3) developmental delay, and at least two minor features among the following: (a) bone age >90th percentile, (b) dysmorphic craniofacial features and (c) congenital anomalies or malformations. These patients were classified in two groups:

- Group I (48 cases) included patients with a recognizable overgrowth condition. Among this group, 31 were classified as patients with Sotos syndrome because they fulfilled the diagnostic criteria defined by Cole and Hughes. ${ }^{7}$ Five were considered as patients with Sotos-like syndrome because they presented with the specific facial gestalt and macrocephaly, but lacked one major criterion, namely, advanced bone age and/or overgrowth. Five presented the suggestive facial gestalt, overgrowth, macrocephaly, camptodactyly and accelerated skeletal maturation characteristic of Weaver syndrome. ${ }^{8,9}$ Two patients with macrosomia, macroglossia, midline abdominal wall defects and neonatal hypoglycemia were diagnosed as having the Beckwith-Wiedemann syndrome. ${ }^{10,11}$ The diagnosis of macrocephaly-cutis marmorata telangiectatica congenita syndrome was established in three patients with skin abnormalities. ${ }^{12}$ One patient presented with suggestive facial gestalt (shallow orbits), accelerated growth and skeletal maturation, overgrowth and broad middle phalanges characteristic of the Marshall-Smith syndrome. ${ }^{13}$ One patient was considered as having the Simpson-GolabiBehmel syndrome, because he presented with macrocephaly, the characteristic facial dysmorphism (large protruding jaw, broad nasal bridge), supernumerary nipples, rib abnormalities, hepatosplenomegaly and cardiac abnormalities. ${ }^{14}$

- Group II (45 cases) included patients with unclassified overgrowth syndromes.

All patients had a normal high-resolution karyotype with no evidence of unbalanced rearrangement. In both groups, a mutation within the NSD1 and GPC3 genes was excluded by direct DNA sequencing, as well as 11 p15 region anomalies. Finally, fluorescence in situ hybridization (FISH) analysis was performed to exclude a deletion involving the 5q35 (NSD1 gene), $9 \mathrm{q} 22$ and 22q13.3 regions and a duplication of the $15 \mathrm{q} 26.3$ and 4 p16.3 regions.

Blood samples were obtained from the probands and their parents after informed consent. Genomic DNA was isolated from blood leukocytes using a Nucleon kit (GE Healthcare, Amersham Biosciences, Piscataway, NJ, USA) according to the manufacturer's instructions.

\section{Array-CGH}

The microarray used in this study is the CytoChips microarray (BlueGnome Ltd, Cambridge, UK). These arrays are spotted with 4400 clones spread over the entire genome with a mean resolution of one clone per megabase. Subtelomeric regions and most of the characterized microdeletion syndromes are included in these clones. All clones are set in duplicate. Array hybridization was undertaken according to the manufacturer's recommendations. After hybridization, slides were scanned on a GenePix 4000B scanner (Molecular Devices, Sunnyvale, CA, USA). Fluorescent intensities were extracted and analyzed using BlueFuse software (BlueGnome, Cambridge, UK). Data were normalized by dividing the mean ratio of each clone duplicate by the median ratio of all autosomal clones. Clones were excluded in cases in which duplicate values differed from each other by more than $10 \%$. A clone was considered to be positive for duplication or deletion if the log2 fluorescence intensities ratio exceeded a value of the mean plus or minus four times the standard deviations (SDs) beyond the mean ratio. Agilent $244 \mathrm{~K}$ oligonucleotide microarrays (Agilent Technologies, Santa Clara, CA, USA) were used according to the manufacturer's recommendations and provide a mean resolution of $20 \mathrm{~kb}$.

\section{Fluorescence in situ hybridization}

BACs were selected from several databases (University of California, Santa Cruz http://genome.ucsc.edu/ and National Center for Biotechnology Information http://www.ncbi.nlm.nih.gov/). FISH studies were performed using standard protocols. ${ }^{15}$

\section{RESULTS}

\section{Molecular karyotyping and FISH analyses}

A total of 93 patients, 68 males and 25 females, who presented with syndromic overgrowth were studied using array-CGH on a BAC/PAC microarray at a mean $1-\mathrm{Mb}$ resolution. To identify potentially clinically relevant anomalies from benign chromosomal variants, we first took advantage of the Database of Genomic Variants (http:// projects.tcag.ca/variation/) to exclude copy number variations (CNVs) previously observed in control individuals. Ten chromosome gains or losses detected in nine patients were thereby considered as being possibly pathogenic and were investigated further. Among these, four are deletions and six are duplications. All imbalances mapped to distinct nonoverlapping regions.

All potentially pathogenic imbalances were confirmed by FISH analysis. Patient 4 was found to carry an amplification of the $18 \mathrm{q} 21.3$ region. FISH studies performed on nuclei revealed - four to five hybridization signals. Retrospectively, the deletion of case 5 was visible on a high-resolution karyotype.

To further show the clinical relevance of these chromosomal aberrations, we tested clinically normal parents by FISH analysis to discriminate between de novo or inherited anomalies. Three CNVs were inherited from a phenotypically normal parent and were interpreted as likely benign variants with no clinical significance. One patient carries an 8q23.3 duplication inherited from a healthy father. Another case carries two chromosomal abnormalities (a 2q22.1 deletion and a 10p13 duplication) both inherited from his healthy father. Five rearrangements (patients 3-7) occurred de novo and are likely pathogenic. The two remaining imbalances are X-linked CNVs (an Xq28 duplication (case 1) and an Xp22.31 deletion (case 2)) inherited from a healthy mother. The mother of patient 1 showed a completely skewed X-inactivation pattern, supporting the potential pathogenicity of this CNV. Unfortunately, the DNA sample from the mother of patient 2 was not available to study the X-chromosome-inactivation pattern. The array-CGH profiles of these cases are shown in Figure 1.

Finally, high-resolution microarray analysis was performed to precisely define the size of the rearrangements. The size of the aneusomic segment varied from 0.32 to $18.2 \mathrm{Mb}(0.32-3.74 \mathrm{Mb}$ for duplications and $1.58-18.2 \mathrm{Mb}$ for deletions).

These seven cases are summarized in Table 1 and have been submitted to the DECIPHER database (http://www.sanger.ac.uk/ PostGenomics/decipher/). This provides access to detailed phenotype information and allows to view the genomic imbalances within the context of the genome browser, Ensembl (http://www.ensembl. org/index.html).

\section{Clinical data}

The clinical features of patients with clinically relevant chromosomal abnormalities are described below.

Patient 1. Patient 1 was ascertained at 7 years of age because of developmental delay and dysmorphism. His birth weight was $2840 \mathrm{~g}$ (50-10th centile), length was $50 \mathrm{~cm}$ (50th centile) and head circumference was $35 \mathrm{~cm}$ (50th centile). He began to sit at 2 years and walk at 5 years. At 7 years, speech is absent, in contrast to his ability to understand. Some stereotypies were also noted. There was no history 

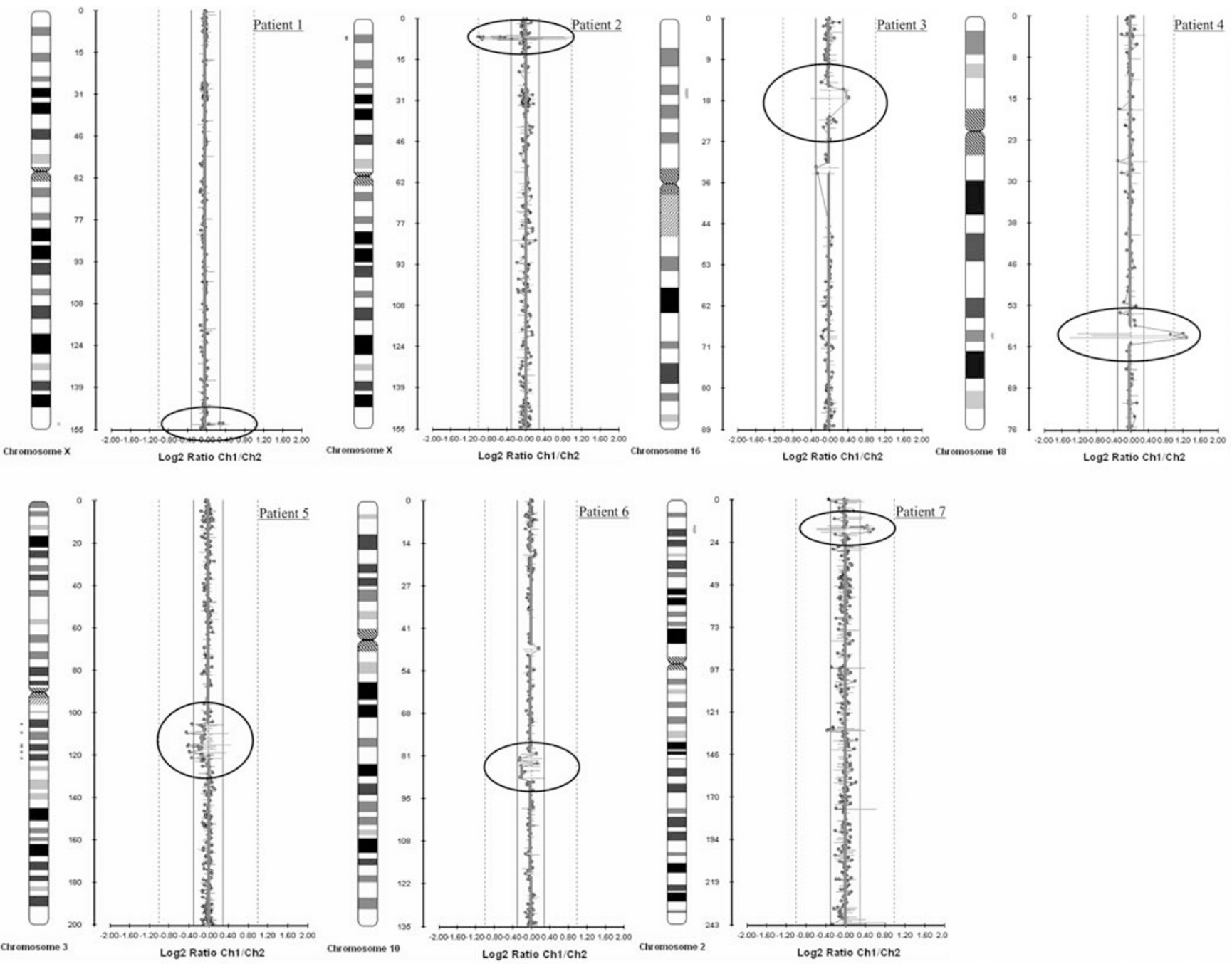

Figure 1 Array-based comparative genomic hybridization ratio profiles showing chromosomal imbalances. For each graph, the $y$ axis marks the distance from the telomere of the short arm (Mb) and the $x$ axis marks the hybridization ratio represented on log2. Vertical lines indicate the threshold for clone deletion or duplication (mean (4SD)). Aberrant clones are surrounded.

Table 1 Patients with possibly pathogenic chromosomal anomalies

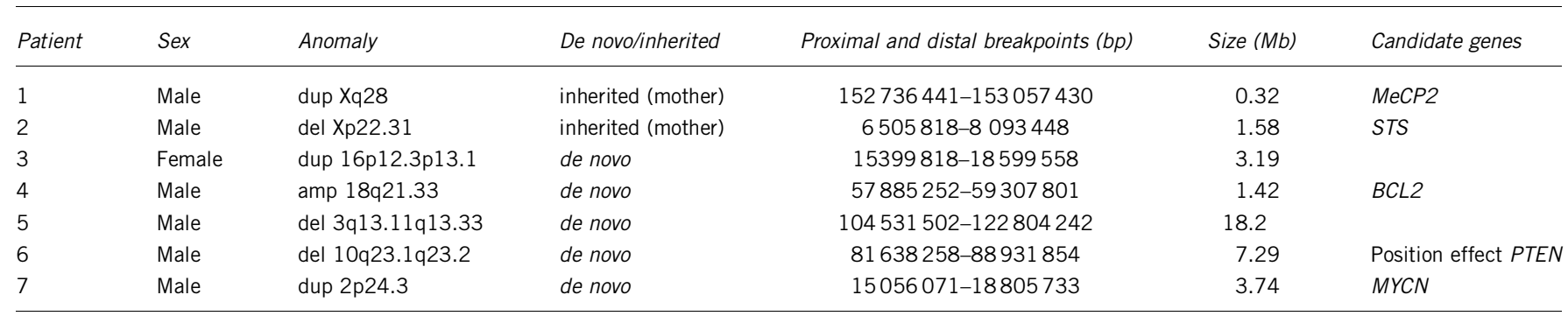

Abbreviations: Del, deletion; dup, duplication.

Data obtained from micro-array Agilent $244 \mathrm{~K}$.

of feeding problems or infectious diseases. His physical examination showed macrocephaly $>+4 \mathrm{SD}(58.5 \mathrm{~cm})$ with height $125 \mathrm{~cm}(+1 \mathrm{SD})$ and weight $26.6 \mathrm{~kg}(+1 \mathrm{SD})$. His mother and father were 150 and $168 \mathrm{~cm}$ in height, respectively. Severe hypotonia was noted.
Dysmorphic features include microstomia and large ears. He displayed livedo and contractures of several joints such as of the knees and elbows. This patient carries a 320-kb-long Xq28 duplication inherited from his healthy mother. 
Patient 2. The propositus was born at 38 weeks of gestation after an uneventful pregnancy with birth parameters above the mean (weight $3900 \mathrm{~g}$ (50-90th centile), height $55 \mathrm{~cm}$ ( $>95$ th centile) and head circumference $41.5 \mathrm{~cm}$ ( $>95$ th centile)). In early infancy, he showed developmental delay. He began to walk at 22 months and he had a speech delay. At 6 years of age, he had a height of $120 \mathrm{~cm}(+1.2 \mathrm{SD})$, a weight of $29 \mathrm{~kg}(+3 \mathrm{SD})$ and a head circumference of $57 \mathrm{~cm}$ $(>+4 \mathrm{SD})$. His mother and father were 168 and $183 \mathrm{~cm}$ in height, respectively. Clinical examination revealed skin abnormalities suggesting ichthyosis. The diagnosis of ichthyosis has also been proposed in the mother and grandfather. Cerebral resonance magnetic imaging showed mild ventricular asymmetry. This patient carries a $1.58-\mathrm{Mb}-$ long Xp22.31 deletion inherited from his healthy mother.

Patient 3. Patient 3 is an 11-year-old girl born at term with a weight of $3510 \mathrm{~g}$ (50-90th centile), a height of $49 \mathrm{~cm}$ (50th centile) and a head circumference of $37 \mathrm{~cm}(>2 S D)$. In the neonatal period, she had poor sucking and abnormal movements requiring medicine (Depakine). In early infancy, severe developmental delay was noted. She was able to hold her head at 9 months and began to walk at 5 years. She said only a few words at the age of 9 years and 6 months. Some stereotypies were also noted. On examination at 11 years of age, growth parameters were weight $33 \mathrm{~kg}(\mathrm{M})$, length $153 \mathrm{~cm}(+2 \mathrm{SD})$ and head circumference $55.5 \mathrm{~cm}(+2 \mathrm{SD})$. Her mother and father were 163 and $172 \mathrm{~cm}$ in height, respectively. She had dysmorphic facial features including a high forehead, a thin and long face, a narrow mandible, a short philtrum and prominent upper incisors. Deformity (genu valgum) in the lower limbs was observed. This patient carries a 3.19-Mb-long $16 \mathrm{p} 12.3 \mathrm{p} 13.1$ de novo duplication.

Patient 4. Patient 4 was born at term with birth parameters in the normal range (weight $3180 \mathrm{~g}$ (50th centile), height $49.5 \mathrm{~cm}$ (50th centile) and head circumference $36.5 \mathrm{~cm}$ (50-90th centile)). Hypotonia and psychomotor development delay were noted. He held his head at 4 months, sat at 15 months and walked without help at 22 months. He had a speech delay. In the first years of life, he presented with feeding problems. He underwent surgery because of phimosis. On physical examination at the age of 3 years and 6 months, his length was $100 \mathrm{~cm}$ (+1SD), height was $17.5 \mathrm{~kg}(+3 \mathrm{SD})$ and head circumference was $54 \mathrm{~cm}$ (>2SD). His mother and father were 160 and $179 \mathrm{~cm}$ in height, respectively. Dysmorphic facial features included upslanting palpebral fissures, a small nose and a carp-shaped mouth. Ophthalmologic examination revealed strabismus. Triceps spasticity was noted. This patient carries a 1.42-Mb-long 18q21.33 de novo amplification.

Patient 5. Patient 5 was born prematurely at 33 weeks of gestation with a birth weight of $1940 \mathrm{~g}$ (50th centile), length of $45 \mathrm{~cm}$ (50th centile) and head circumference of $32 \mathrm{~cm}$ (50th centile). At the age of 3 years, he could walk without help and spoke a few words. On examination, his height was $119 \mathrm{~cm}$ (>2SD), his weight was $19 \mathrm{~kg}$ $(>2 S D)$ and his head circumference was $53 \mathrm{~cm}(+2 \mathrm{SD})$. His mother and father were 170 and $187 \mathrm{~cm}$ in height, respectively. He displayed facial dysmorphism with epicanthus, a prominent nasal bridge, bulbous nasal tip and everted lips. In addition, the patient presented a double hair whorl and a shawl scrotum. This patient carries an 18.2Mb-long 3q13.11q13.33 de novo deletion.

Patient 6. The propositus was born at 38 weeks of gestation through spontaneous vaginal delivery. At birth, weight was $4030 \mathrm{~g}$ ( $>95$ th centile), length was $55 \mathrm{~cm}$ (>95th centile) and head circumference was $35 \mathrm{~cm}$ (50-90th centile). In early childhood, developmental delay was noted. At physical examination at the age of 12 years, his length was $173.5 \mathrm{~cm}(+3 \mathrm{SD})$, weight was $63 \mathrm{~kg}(+4 \mathrm{SD})$ and head circumference was $58 \mathrm{~cm}(+3 / 4 \mathrm{SD})$. His mother and father were $173 \mathrm{~cm}$ and $183 \mathrm{~cm}$ in height, respectively. He presented with speech delay. He had dysmorphic features including dolichocephaly, upslanting palpebral fissures, hypertelorism and retrognathia. A single flexion crease of the hands and a lumbar hyperlordosis were noted. This patient carries a 7.29 Mb-long 10q23.1q23.2 de novo deletion.

Patient 7. This patient was referred to a medical center because of overgrowth and thumb abnormalities. He was born at 38 weeks of gestation after an uneventful pregnancy with a weight of $4270 \mathrm{~g}$ (90th centile), a height of $52 \mathrm{~cm}$ (90th centile) and a head circumference of $37.5 \mathrm{~cm}$ (>95th centile). Triphalangy of the thumbs with clinodactyly was noticed. In early childhood, he presented with a mild developmental delay. He started walking at 18 months and had some language difficulties. At the age of 6 years and 6 months, he attended a regular elementary school with speech therapy once a week. On examination, he had a weight of $35 \mathrm{~kg}$ ( $>3 S \mathrm{SD})$, a height of $133.5 \mathrm{~cm}(>3 \mathrm{SD})$ and a head circumference of $61 \mathrm{~cm}(>3 S D)$. His mother and father were 172 and $180 \mathrm{~cm}$ in height, respectively. He had a facial dysmorphism reminiscent of Weaver syndrome. A lumbar cutaneous angioma and cafe-au-lait spots were noted. He also displayed a moderate pectus excavatum. This patient carries a $3.74-\mathrm{Mb}-$ long $2 \mathrm{p} 24.3$ de novo duplication.

\section{DISCUSSION}

Although some patients with syndromic overgrowth might have been subjected to array-CGH testing in the course of the systematic study of mentally retarded cases, we present in this study the first analysis using whole-genome array-CGH focused on such patients. A total of 93 patients were investigated and 7 potentially pathogenic chromosome imbalances were identified, providing a diagnosis yield of $7.5 \%$. No recurrent abnormality was identified in this cohort and all but one aneusomic fragment have a size smaller than $5 \mathrm{Mb}$. Our results provide further demonstration for the extreme genetic heterogeneity of these conditions. They also suggest that array-CGH should be considered as an essential aspect of the genetic analysis of patients with localized or generalized syndromic overgrowth.

In two cases, however, the clinical relevance of the chromosomal anomaly remains uncertain. This is the case for patient 3 in whom a 16 p13.11 duplication was observed. Recently, Hannes et al ${ }^{16}$ described reciprocal $1.7 \mathrm{Mb}$ deletions and duplications of this region resulting from nonallelic homologous recombination (NAHR) between distinct LCR16 subunits. Although deletion is a risk factor for MR/MCA, the duplication may be a rare benign variant. The duplication observed in patient 3 occurred de novo but is identical to those reported by Hannes et al, ${ }^{16}$ making its clinical interpretation difficult.

Similarly, the clinical relevance of the Xp22.31 microdeletion detected in patient 2 remains questionable. This $1.5-\mathrm{Mb}$ interstitial deletion encompasses the STS (steroid sulfatase) gene and was inherited from a mother with normal intelligence. STS deletion is known to be associated with X-linked ichthyosis (XLI), a feature observed in our patient. By contrast, overgrowth has not been described in males with XLI. High-resolution array-CGH showed that patient 2 carried the recurrent $1.5-\mathrm{Mb}$ deletion, because of NAHR between duplicons, and is observed in $90 \%$ of XLI patients. ${ }^{17}$ Presumably, this anomaly may account for the mental handicap and ichthyosis observed in patient 2; however, whether macrocephaly is linked to this deletion remains unanswered.

Five chromosomal abnormalities identified are clearly pathogenic and allow to draw some interesting conclusions. First, although 
chromosomal deletions are usually associated with growth retardation, three out of five patients have a duplication. Statistical analyses cannot be performed on such a small series; however, our results might therefore suggest that increased gene dosage could be responsible for a significant number of overgrowth cases.

Second, the identification of a $0.32-\mathrm{Mb}$ Xq28 duplication including the $\mathrm{MeCP} 2$ (methyl-CpG-binding protein 2) and L1CAM genes in patient 1 provides further evidence of the phenotypic variability of $\mathrm{MeCP} 2$ duplications. Males with an $\mathrm{MeCP} 2$ duplication manifest infantile hypotonia, severe mental retardation, absence of speech, neurological problems (progressive spasticity and seizures) and recurrent respiratory infections, ${ }^{18}$ whereas growth parameters, including head circumference, are usually within the normal range. Our patient does not appear to fulfill this common phenotype associated with Xq28 duplication. Interestingly, two recent reports describe the association of $\mathrm{MeCP} 2$ duplication with macrocephaly. ${ }^{19,20}$ We therefore suggest that severe psychomotor delay, absence of speech and macrocephaly in males may point to the screening for $\mathrm{MeCP} 2$ duplication, even in the absence of recurrent respiratory infections.

Finally, three aneusomic segments identified in this study involved oncogenes or tumor-suppressor genes. The MYCN gene maps within the 2 p24.3 duplicated segment observed in patient 7. Overexpression of $\mathrm{dMyc}$, the drosophila Myc ortholog, promotes cell growth and induces apoptosis in adjacent cells expressing lower $\mathrm{dMyc}$, and larger wings are observed on ubiquitous overexpression of $\mathrm{dMyc}$ during wing development. ${ }^{21,22}$ Moreover, in humans, haploinsufficiency of the MYCN gene is responsible for Feingold syndrome. This disorder is characterized by intestinal atresias, digital anomalies, facial dysmorphism and learning disability. Microcephaly is one of the most common features present in about $90 \%$ of patients with Feingold syndrome. ${ }^{23}$ Altogether, these data are consistent with a function of MYCN overexpression in the etiology of the overgrowth observed in patient 7. Similarly, we observed a 4-5 times amplification of the genomic segment encompassing the BCL2 gene in patient 4 . The translocation between chromosomes 14 and 18 (ie, $\mathrm{t}(14 ; 18)(\mathrm{q} 32.3 ; \mathrm{q} 21)$ ) is the hallmark of follicular B lymphomas. This chromosomal rearrangement leads to a fusion between an IgH enhancer and BCL2, resulting in the overexpression of the antiapoptotic BCL2 protein leading to uncontrolled cell proliferation. Noticeably, transgenic mice overexpressing human BCL2 protein in neurons underwent nervous system hypertrophy and reduced neuronal loss during naturally occurring cell death. ${ }^{24}$ Finally, patient 6 carries a 10q23.1q23.2 deletion, the distal break point of the rearrangement of which lies close to the PTEN gene $(0.68 \mathrm{Mb})$. Deletions and mutations of PTEN have been encountered in various hamartoma-overgrowth syndromes, collectively designated as 'PTEN hamartoma tumor syndromes. ${ }^{25,26}$ Recently, it has been shown that germ-line mutations of PTEN can also determine syndromes characterized by autism associated with macrocephaly. ${ }^{27,28}$ Presumably, the haploinsufficiency of genes contained within the 10q23.1q23.2 deletion is responsible for some of the features observed in patient 6 , such as lumbar hyperlordosis or facial dysmorphy. The deletion may also exert a position effect on PTEN gene expression resulting in overgrowth.

Altogether, these data provide further evidence that constitutional oncogene duplications or tumor-suppressor gene deletions are involved in the pathogenesis of overgrowth conditions. In addition, it suggests that an increased susceptibility to neoplasia may be suspected in these patients.

In conclusion, our results emphasize the benefits of whole-genome array-CGH for the diagnosis of overgrowth syndromes. Careful clinical examination of patients may allow the delineation of novel clinically recognizable overgrowth conditions. A detailed molecular analysis of the rearranged regions may pave the way for the identification of genes involved in growth regulation. More importantly, these data underscore the crucial need for a specific tumor surveillance program in patients with overgrowth syndromes.

\section{CONFLICT OF INTEREST}

The authors declare no conflict of interest.

\section{ACKNOWLEDGEMENTS}

We express our gratitude to the patients and their families. This study was supported by the Centre National de la Recherche Scientifique, the GIS-Maladies Rares and the Agence de Biomédecine.

1 Partington MW, Fagan K, Soubjaki V, Turner G: Translocations involving 4 p16.3 in three families: deletion causing the Pitt-Rogers-Danks syndrome and duplication resulting in a new overgrowth syndrome. J Med Genet 1997; 34: 719-728.

2 Faivre L, Gosset P, Cormier-Daire V et al: Overgrowth and trisomy 15q26.1-qter including the IGF1 receptor gene: report of two families and review of the literature. Eur J Hum Genet 2002; 10: 699-706.

3 Tatton-Brown K, Pilz DT, Orstavik KH et al: $15 \mathrm{q}$ overgrowth syndrome: a newly recognized phenotype associated with overgrowth, learning difficulties, characteristic facial appearance, renal anomalies and increased dosage of distal chromosome 15q. Am J Med Genet A 2009; 149A: 147-154.

4 Kurotaki N, Imaizumi K, Harada N et al: Haploinsufficiency of NSD1 causes Sotos syndrome. Nat Genet 2002; 30: 365-366.

5 Redon R, Baujat G, Sanlaville D et al: Interstitial 9q22.3 microdeletion: clinical and molecular characterisation of a newly recognised overgrowth syndrome. Eur J Hum Genet 2006; 14: 759-767.

6 Phelan MC, Rogers RC, Saul RA et al: 22q13 deletion syndrome. Am J Med Genet 2001; 101: 91-99.

7 Cole TR, Hughes HE: Sotos syndrome: a study of the diagnostic criteria and natural history. J Med Genet 1994; 31: 20-32.

8 Rio M, Clech L, Amiel J et al: Spectrum of NSD1 mutations in Sotos and Weaver syndromes. J Med Genet 2003; 40: 436-440.

9 Baujat G, Rio M, Rossignol S et al: Clinical and molecular overlap in overgrowth syndromes. Am J Med Genet C Semin Med Genet 2005; 137C: 4-11.

10 Weksberg R, Smith AC, Squire J, Sadowski P: Beckwith-Wiedemann syndrome demonstrates a role for epigenetic control of normal development. Hum Mol Genet 2003; 12 (Spec No 1): R61-R68.

11 Baujat G, Rio M, Rossignol S et al: Paradoxical NSD1 mutations in BeckwithWiedemann syndrome and $11 \mathrm{p} 15$ anomalies in Sotos syndrome. Am J Hum Genet 2004; 74: 715-720.

12 Lapunzina P, Gairi A, Delicado A et al: Macrocephaly-cutis marmorata telangiectatica congenita: report of six new patients and a review. Am J Med Genet A 2004; 130A: 45-51.

13 Williams DK, Carlton DR, Green SH, Pearman K, Cole TR: Marshall-Smith syndrome: the expanding phenotype. J Med Genet 1997; 34: 842-845.

14 Neri G, Gurrieri F, Zanni G, Lin A: Clinical and molecular aspects of the SimpsonGolabi-Behmel syndrome. Am J Med Genet 1998; 79: 279-283.

15 Romana SP, Cherif D, Le Coniat M, Derre J, Flexor MA, Berger R: In situ hybridization to interphase nuclei in acute leukemia. Genes Chromosomes Cancer 1993; 8: 98-103

16 Hannes FD, Sharp AJ, Mefford HC et al: Recurrent reciprocal deletions and duplications of 16p13.11: the deletion is a risk factor for MR/MCA while the duplication may be a rare benign variant. J Med Genet 2009; 46: 223-232.

17 Van Esch H, Hollanders K, Badisco L et al: Deletion of VCX-A due to NAHR plays a major role in the occurrence of mental retardation in patients with $\mathrm{X}$-linked ichthyosis. Hum Mol Genet 2005; 14: 1795-1803.

18 Van Esch H, Bauters M, Ignatius J et al: Duplication of the MECP2 region is a frequent cause of severe mental retardation and progressive neurological symptoms in males. Am J Hum Genet 2005; 77: 442-453.

19 del Gaudio D, Fang P, Scaglia F et al: Increased MECP2 gene copy number as the result of genomic duplication in neurodevelopmentally delayed males. Genet Med 2006; 8: 784-792.

20 Lugtenberg D, Kleefstra T, Oudakker AR et al: Structural variation in Xq28: MECP2 duplications in $1 \%$ of patients with unexplained XLMR and in $2 \%$ of male patients with severe encephalopathy. Eur J Hum Genet 2009; 17: 444-453.

21 de la Cova C, Abril M, Bellosta P, Gallant P, Johnston LA: Drosophila myc regulates organ size by inducing cell competition. Cell 2004; 117: 107-116.

22 Moreno E, Basler K: dMyc transforms cells into super-competitors. Cell 2004; 117: 117-129.

23 Marcelis CL, Hol FA, Graham GE et al: Genotype-phenotype correlations in MYCNrelated Feingold syndrome. Hum Mutat 2008; 29: 1125-1132. 
24 Martinou I, Fernandez PA, Missotten M et al: Viral proteins E1B19K and p35 protect sympathetic neurons from cell death induced by NGF deprivation. J Cell Biol 1995; 128: 201-208.

25 Eng C: PTEN: one gene, many syndromes. Hum Mutat 2003; 22: 183-198.

26 Blumenthal GM, Dennis PA: PTEN hamartoma tumor syndromes. Eur J Hum Genet 2008; 16: 1289-1300.
27 Butler MG, Dasouki MJ, Zhou XP et al: Subset of individuals with autism spectrum disorders and extreme macrocephaly associated with germline PTEN tumour suppressor gene mutations. J Med Genet 2005; 42: 318-321.

28 Orrico A, Galli L, Buoni S, Orsi A, Vonella G, Sorrentino V: Novel PTEN mutations in neurodevelopmental disorders and macrocephaly. Clin Genet 2009; 75: 195-198. 